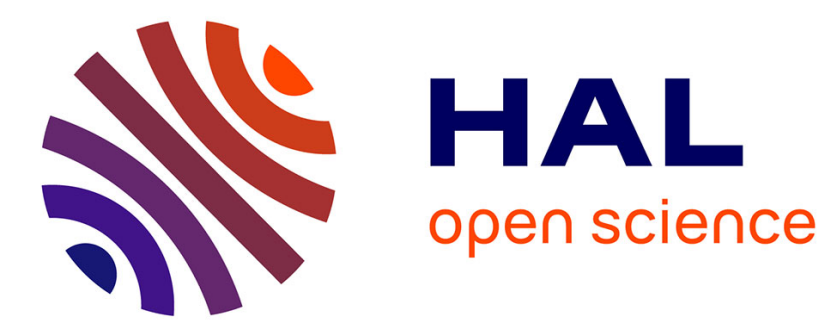

\title{
Philosophie esthétique de l'Inde
}

Nicolas Go

\section{- To cite this version:}

Nicolas Go. Philosophie esthétique de l'Inde. Le Philosophoire, 2014, pp.47-72.

10.3917/phoir.041.0039 . hal-01138644

\section{HAL Id: hal-01138644 \\ https://hal.science/hal-01138644}

Submitted on 2 Apr 2015

HAL is a multi-disciplinary open access archive for the deposit and dissemination of scientific research documents, whether they are published or not. The documents may come from teaching and research institutions in France or abroad, or from public or private research centers.
L'archive ouverte pluridisciplinaire HAL, est destinée au dépôt et à la diffusion de documents scientifiques de niveau recherche, publiés ou non, émanant des établissements d'enseignement et de recherche français ou étrangers, des laboratoires publics ou privés. 


\title{
Philosophie esthétique de l'Inde
}

\section{Nicolas Go \\ Université de Rennes 2}

\begin{abstract}
Résumé
Cette contribution se propose de présenter la manière dont l'Inde a pensé le problème de l'esthétique, à travers le théâtre et la poésie notamment, mais de telle sorte que, aujourd'hui encore, tous les arts s'y reconnaissent. Le texte de référence, le Nātyaśāstra ou Traité du théâtre, attribué à Bharata, bien qu'il hérite d'une tradition plus ancienne, date du début de notre ère ; il a donné lieu, parmi tant d'autres, à un commentaire magistral,l'Abhinavabhāratī, par Abhinavagupta, maître du śivaïsme cachemirien.

L'expérience esthétique, rapprochée de la pratique spirituelle ou mystique, est décrite, conceptualisée et interprétée dans la constante préoccupation de lier la présentation des formes concrètes de sa mise en oeuvre au caractère ineffable de l'expérience, dont l'essence est la joie.
\end{abstract}

L'expérience esthétique a été très tôt pensée en Inde, dans le théâtre qui est un art total comprenant le jeu dramatique, la danse, la musique, la poésie, puis à nouveau dans de nombreux traités sur la poétique ${ }^{1}$, jusqu'à la monumentale synthèse du philosophe tantrique et esthéticien du Cachemire Abhinavagupta $\left(\mathrm{X}^{\circ}-\mathrm{XI}^{\circ}\right.$ siècle), sous l'espèce de son commentaire l'Abhinavabhāratī, qui retiendra ici plus particulièrement notre attention. L'analyse de l'expérience artistique a progressivement conduit à un véritable travail de théorisation et de conceptualisation, empruntant à des domaines divers tout en produisant ses propres catégories originales. A l'écart des traités théologiques ou religieux, les penseurs de l'esthétique ont produit une importante littérature profane, souvent très technique, néanmoins toujours reliée à ce grand projet spirituel qui caractérise la civilisation de l'Inde. Je voudrais ici circonscrire mon propos à l'expérience elle-même, telle qu'elle a été pensée dans quelques grands textes, en tentant à la fois de dessiner le paysage conceptuel de la philosophie esthétique indienne, et de faire comprendre la manière dont celle-ci se retire au seuil d'une expérience spirituelleà laquelle elle introduit, et dont la sagesse constitue le véritable projet.

En raison du caractère hétérogène de la pensée indienne, j'ai choisi d'encombrer mon texte des concepts et éléments de vocabulaire sanskrits que j'estime utiles pour rendre compte de la spécificité du contexte intellectuel et culturel, et éviter ainsi les confusions de traductions jamais satisfaisantes ou les tentationsd'un comparatisme abusif. La lecture s'en trouvera certes malaisée mais, je l'espère, plus précise.

\section{La saveur esthétique}

Le concept central est celui de rasa, qui désigne étymologiquement la sève, le suc, l'essence ou la saveur ${ }^{2}$. Il définit l'expérience esthétique elle-même, conçue comme une forme de connaissance spirituelle autant que comme un acte de jubilation, et plus particulièrement l'essence de la poésie : « le rasa est tout simplement l'essence de la poésie » affirme Abhinavagupta (Gnoli, p. 63). Le poéticien Viśvanātha, auteur du Sāhityadarpaṇa, le

\footnotetext{
${ }^{1}$ Depuis Dandin, VIII ${ }^{\circ}$ s., et tout au long de l'époque médiévale.

${ }^{2}$ Bharata précise que le rasa est « ce qui est susceptible d'être savouré ».
} 
définit comme «fait de pleine Intelligence, Béatitude et Lumière; il est étranger à tout contact avec aucune autre chose connue, frère de l'expérience du brahman. Il est constitué d'un émerveillement (camatkāra) de nature non-ordinaire. Il est éprouvé comme l'unité de notre être même » (Coomaraswamy, p. 48). Dans la tradition indienne, l'expérience esthétique manifeste un état d'émerveillement d'une nature spécifique, celle d'une béatitude supérieure à toute expérience ordinaire de plaisir ou de joie. Mieux encore, si le rasa est l'essence de l'art ajouteAbhinavagupta, la béatitude est l'essence du rasa: «car l'essence de la lumière [du rasa] - une lumière extrêmement dense, consistant en la délectation de la conscience par ellemême - est la béatitude. [...] Ce qui est savouré est la seule conscience, saturée de béatitude » (Gnoli, p. 87).

Pour bien saisir ce concept de rasa, il convient de le situer dans son contexte source, tel qu'il a été formalisé dans le Nātyaśāstra, et défini au sixième chapitre dans un aphorisme inaugural, qui a fait l'objet de très nombreux commentaires : «Le rasa naît de la conjonction des déterminants (vibhāva), des conséquents (anubhāva) et des dispositions d'esprit temporaire (vyabhicāribhāva) ». L'enjeu est ici d'expliciter cette expérience esthétique dans le théâtre, laquelle se distingue de tout ce dont on peut faire communément l'épreuve. En ce sens, la délectation est reconnue de caractère non-ordinaire, " supra-mondain » (alaukika), et nécessite des concepts spécifiques : les déterminants et les conséquents sont les analogues respectifs des causes et des effets ${ }^{3}$ de la vie ordinaire (laukika), qui, du fait de la situation théâtrale, contribuent à l'apparition de dispositions d'esprit de nature émotionnelle et passagère, déterminées par ces éléments artistiques. Ces facteurs actualisent des « dispositions d'esprit permanentes » (sthāyibhāova), au nombre de huit, présentes comme potentialités incapables de se manifester d'elles-mêmes. Ces dispositions d'esprit permanentes relèvent de ce que la psychologie indienne conçoit comme «impressions résiduelles dynamiques » (samskāra), dispositions acquises par l'expérience de la vie réelle (y compris la fréquentation des œuvres littéraires ou des spectacles) et comme «imprégnations » (vāsanā) déposées dans le psychisme au cours des vies antérieures, persistant inconsciemment et à l'état latent, à la manière d'une odeur de parfum tenace dans un flacon vide. Dans la vie commune, ces dispositions permanentes donnent naissance aux émotions et aux sentiments ordinaires ; mais déterminées par les trois facteurs de l'art théâtral combinés, elles s'actualisent sous la figure du rasa. De même que le convive apprécie la saveur d'un mets issue de la combinaison des épices, herbes et ingrédients, l'esthète jouit de la saveur de l'œuvre née de la combinaison des éléments spécifiques à l'art dramatique.

Bharata explique ce phénomène jubilatoire chez l'individu raffiné par l'existence des sthāyibhāva: la manifestation théâtrale des trois facteurs produit chez le spectateur l'émergence d'une saveur esthétique, à partir de dispositions affectives inconscientes. Il convient donc de bien distinguer d'une part les conditions de possibilité artistiques et matérielles, et d'autre part celles, psychiques, de l'expérience esthétique.

\section{a-Les facteurs artistiques :}

- Les déterminants ou vibhāva désignent toute cause induisant un état physique, émotionnel ou mental particulier ; ce sont notamment les personnages (humains ou animaux) et les circonstances scéniques, telles qu'une saison plaisante, des guirlandes de fleurs, des parcs et jardins, des ornements, etc.

\footnotetext{
${ }^{3}$ karana et kārya.
} 
- les conséquents ou anubhāva désignent toutes les gestuelles expressives des acteurs ; ainsi les mouvements gracieux du corps, les sourires charmants, les mots plaisants, les regards signifiants, les figures de danse, etc. ${ }^{4}$

- Les dispositions d'esprit temporaires ou vyabhicāribhāva sont les sentiments ordinaires théâtralisés par la situation dramatique elle-même ; ils imitent ceux de la vie réelle (bhāva), comme le délice, l'excitation, la rêverie, l'étonnement, la jalousie, etc. ${ }^{5}$

Savamment associés, ces facteurs artistiques actualisent certaines dispositions d'esprit permanentes comme, pour les exemples cités ci-dessus, le délice amoureux dans l'union. Elles «s'universalisent» alors spontanément et simultanément en rasa, ici l'Amoureux (śrngāra), pour former l'expérience esthétique.

\section{$b-L$ 'expérience esthétique :}

- Les dispositions d'esprit permanentes(sthāyibhāva) qui constituent, on l'a évoqué, le substrat latent des rasa, sont au nombre de huit: le délice (rati), la gaieté (hāsa), la peine (śoka), la colère (krodha), la fougue (utsāha), la peur (bhaya), l'aversion (jugupsāa) et la surprise (vismaya). Elles représentent la totalité des mouvements affectifs de la conscience humaine ${ }^{6}$.

- Les rasa (saveurs esthétiques) entretiennent avec elles une correspondance terme à terme, respectivement l'Amoureux (śrñgāra), le Comique (hāsya), le Pathétique (karuna), le Furieux (raudra), l'Héroïque (vīra), le Terrible (bhayānaka), l'Odieux (bībhatsa) et le Merveilleux (adbhuta). Ils sont «ce qui est susceptible d'être savouré », d'après Bharata, l'essence même de l'expérience esthétique, et également la visée pratique de l'artiste.

Mais quelle est l'unité fonctionnelle et synthétique de cet ensemble, et son intérêt problématique pour nous?

Si le doublet sthāyibhāva et rasa constitue un référent conceptuel pour l'ensemble des théories esthétiques en Inde, en revanche, les «facteurs artistiques », parce qu'ils sont nés de la théorisation particulière de la pratique théâtrale, ne peuvent tenir lieu de schème explicatif pour tout art en général. Associés à la production concrète de l'œuvre, ils se spécifient selon les arts. Les poéticiens sont les seuls à avoir tenté, pour le langage, une nouvelle analyse portant sur les conditions du procès d'épanouissement du rasa. Pour les autres disciplines

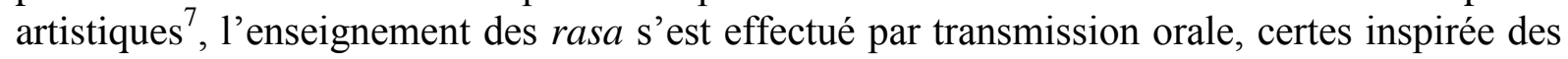
textes, mais toujours associé à la pratique elle-même.

La thèse en poétique est la suivante : les mots sont dotés d'un "pouvoir » propre (vyāpāra) de manifester le rasa, doublement déterminé par la puissance créatrice du poète ${ }^{8}$ et la sensibilité du lecteur (ou de l'auditeur) ${ }^{9}$. Le rasa, selon Ānandavardhana ${ }^{10}$, est conçu

\footnotetext{
${ }^{4}$ Bharata mentionne également les sattvikabhāva, mouvements émotionnels soudains au nombre de huit, de nature réactive et physiologique, produits, à partir des traces psychiques latentes, par effort de concentration des acteurs, et qui ont pour vocation d' «imiter la nature humaine » afin de persuader par réalisme; ainsi par exemple les larmes, la transpiration, le rougissement, les tremblements du corps, etc. (Bharata, 1988, pp. 92105).

${ }^{5}$ Ces bhāva sont au nombre de trente-trois (ibid. pp. 27-92).

${ }^{6}$ On lira avec intérêt les travaux de Mikel Dufrenne (1953/1992), qui tented'établir une thèse des a priori affectifs en écho à la première critique de Kant [quatrième partie « critique de l'expérience esthétique », chap. premier (Les a priori affectifs) et deuxième (La connaissance a priori des a priori affectifs et la possibilité d'une esthétique pure)].

${ }^{7}$ L'ouvrage illustré de Tara Michaël (1985), propose une description des techniques expressives selon la danse indienne, susceptible de contribuer à une compréhension des « facteurs artistiques » dans cette discipline.

${ }^{8}$ pratibhā.

9 sahrdaya.

${ }^{10} \mathrm{Il}$ est l'auteur de l'ouvrage Dhvanyāloka $\left(\mathrm{IX}^{\circ} \mathrm{s}\right.$.) où apparaît pour la première fois la théorie du dhvani, théorie de l'expression qui a trouvé appui dans la théorie du sphoṭa (voir à ce sujet par exemple l'article de Pierre-
} 
comme une forme de résonance, ou de son suggéré, qui est évoqué plutôt qu'exprimé ; ce pouvoir d'évocation de la poésie provoque, à partir de la simple compréhension des mots lus ou entendus, un passage instantané à l'expérience esthétique, le rasadhvani, qui transcende la pensée discursive et se situe hors du domaine du langage. Bhatta Nāyaka distingue trois pouvoirs des mots : pouvoir de dénotation ${ }^{11}$ qui se réfère au sens et qui est commun à toute forme d'écrit, pouvoir de révélation ${ }^{12}$ qui consiste en la manifestation ou l'évocation du rasa, et pouvoir de jubilation esthétique ${ }^{13}$, ces deux derniers étant propres à la poésie. Le pouvoir de révélation, explique Abhinavagupta, se définit par la faculté propre au texte poétique, d'universaliser - sublimer - les facteurs artistiques (déterminants, etc.) ; hors la référence au théâtre, les moyens de la poésie se définissent par un ensemble de caractéristiques qui se rassemblent sous trois titres : l'absence de défauts ${ }^{14}$, la présence de qualités ${ }^{15}$ et l'existence d'ornements ${ }^{16}$. Il a également la faculté de supprimer la stupeur mentale qui enchaîne, asservit la conscience dans l'existence ordinaire.

En somme, il consiste, au moyen de techniques d'écriture élaborées, à délivrer la conscience de son mode de pensée qui reste inscrit dans les représentations mentales, particulièrement celles d'espace et de temps, pour la faire accéder spontanément et simultanément à la lecture, à une expérience singulière de jubilation. Cette jubilation, ou délectation, est en tout étrangère aux plaisirs de la vie commune, lorsque le psychisme reste attaché à des expériences et des formes de perception décrites en Inde par la Logique et les théories de la connaissance (raisonnement, analogie, etc.); elle surgit des diverses corrélations de ces qualités immanentes de la Nature que sont les guna, qui se réorganisent en mouvements de conscience dits d'expansion ${ }^{17}$, de fluidité ${ }^{18}$, et de dilatation ${ }^{19}$. La jubilation se caractérise, du fait de la domination de sattva, la qualité la plus spirituelle (luminosité, transparence, joie, sérénité, clairvoyance), par ce qu'on pourrait nommer un repos de la conscience en elle-même: ceci exprime un état de totale immersion dans l'objet de la délectation, exclusif de toute préoccupation extrinsèque, de tout désir, de toute crainte ; sous la plume d'un philosophe tantrique, la conscience n'est pas la simple possibilité de faire retour sur soi-même et qui fait de l'homme un sujet capable de penser le monde, elle désigne la Conscience absolue, «le réceptacle du grand Vide » qui, « réfléchissant sur elle-même, se perçoit comme distincte de toute l'objectivité en se disant: je ne suis pas cela (neti neti) ${ }^{20}$, l'état le plus élevé auquel atteignent les yogin; elle est Acte pur, vacuité vide de tout substrat, de toute catégorie et des sédiments des actes, liberté, ce «vide interstitiel » dont Jayaratha, glosant Abhinavagupta, dit: «c'est lui qu'on nomme vacuité et lieu de repos ${ }^{21}$. C'est pourquoi la délectation dans l'œuvre a en commun avec la félicité du yogin l'expérience

Sylvain Filliozat, «le Sphoța», Les Cahiers de Philosophie, n 14, 1992, pp. 155-169); la théorie de l'expression distingue la dénotation directe, l'indirecte ou induite (métaphore) et le sens suggéré. Le dhvani se laisse décrire selon l'élément qui le reçoit, sa relation avec le sens direct, et selon sa nature (qu'il porte sur un objet, une figure rhétorique ou un sentiment). Mammața $\left(\mathrm{XI}^{\circ} \mathrm{s}\right.$.) hiérarchise la valeur des textes poétiques selon le critère du dhvani : la poésie par excellence est celle où il règne totalement, la poésie inférieure celle où il existe à l'état subordonné, et la poésie basse celle où il fait défaut.

${ }^{11}$ abhidhā.

${ }^{12}$ bhāvanā.

${ }^{13}$ bhogì karana.

${ }^{14}$ doṣa.

${ }^{15}$ guna.

${ }^{16}$ alàikāra.

${ }^{17}$ vikāsa, provoqué par la prédominance de sattva.

${ }^{18} d r u t i$, déterminé par l'association de sattva et rajas.

${ }^{19}$ vistara, déterminé par l'association de sattva et tamas.

${ }^{20}$ Abhinavagupta, cité dans Lilian Silburn (1983), p. 54.

${ }^{21}$ idem. 
possible de l'absolu, du «brahman suprême ». Ce qui est ainsi goûté ${ }^{22}$ a pour essence la lumière $^{23}$ et la béatitude, ānanda.

\section{Le pouvoir de révélation}

La thèse est ainsi formulée : le matériau de l'art (les mots en poésie et en dramaturgie précise Abhinavagupta, mais aussi bien les notes en musique, les gestes en danse, etc.) est investi d'un pouvoir propre ${ }^{24}$, intuitif et inouï, de « révélation ${ }^{25} \mathrm{~d}$ 'affects esthétiques ${ }^{26}$, qui s'effectue en un mouvement de sublimation ${ }^{27}$ dégagé de toute représentation mentale et de toute conscience individualisante de soi; il en résulte une expérience de jubilation ${ }^{28}$ prodigieuse où la conscience, délivrée de la stupeur ${ }^{29}$ qui caractérise son ordinaire, repose en elle-même, en son essence qui est «lumière ${ }^{30}$ et «béatitude ${ }^{31}$.

La notion de «pouvoir » de révélation de l'œuvre se justifie par l'emprunt explicite du concept de rasa à l'Atharvaveda, ${ }^{32}$ celui des quatre Veda le plus attaché à l'efficace de l'action rituelle. Abhinavagupta compare dans son commentaire les phénomènes esthétiques dans la représentation théâtrale à ceux, magiques, dans les rites propitiatoires des officiants védiques: à l'instar de l'accomplissement du rituel sacrificiel, où gestes, paroles et chants,strictement ordonnés, s'accompagnent d'effets magiques, la création dans l'art, rigoureusement élaborée, est porteuse d'un pouvoir qui transcende les moyens mis en œuvre. Ce pouvoir détourne la conscience des fins pratiques ordinaires, qui soumettent la perception à des représentations hétérogènes, secondes par rapport à la perception elle-même : percevoir un ordre, formuler un désir ou une intention, puis s'exécuter. Limitée par le choix sélectif de la perception en fonction de la représentation d'un acte à accomplir ou d'un intérêt à satisfaire, la conscience se coupe de tout le champ de réalité qui échappe à son projet; elle reste, disent les auteurs, asservie par les limitations du temps, de l'espace et de la causalité. Étrangère à elle-même, aliénée.

En revanche, en vertu du pouvoir révélateur de l'œuvre, l'expérience esthétique s'éprouve comme un retour de la conscience à son unité originelle, non limitée par les préoccupations utiles et «mondaines », rendue à la plénitude d'elle-même, dans le seul présent de la délectation : libérée notamment des entraves du temps, de l'idée du passé et du futur, " une telle délectation est comme une fleur née par magie ; son essence est le seul présent ${ }^{33}$. Plus encore, le présent de l'expérience esthétique est vécu sur le mode de l'éternité, «pas plus lié à ce qui est survenu avant qu'à ce qui adviendra après, cette délectation du rasa est, dès lors, aussi différente que celle des yogin à l'égard des plaisirs ordinaires ${ }^{34}$. Ainsi, la bhāvanā n'est pas un simple pouvoir de remémoration, de reproduction et de réitération, dont parlent par exemple les textes de l'école des Vaiśeșika, de rendre à nouveau présent le passé à partir des impressions résiduelles ${ }^{35}$; elle n'appartient pas à

\footnotetext{
${ }^{22} \bar{a} \operatorname{sva} d a$

${ }^{23}$ prakāśa

${ }^{24}$ vyāpāra

${ }^{25}$ bhāvanā

${ }^{26}$ rasa

${ }^{27}$ sādhārana

${ }^{28}$ bhoga

${ }^{29}$ moha

${ }^{30}$ prakāía

${ }^{31} \bar{a}$ nanda

${ }^{32}$ «Il prit les paroles au Rgveda, la musique au Sämaveda, les techniques de jeu au Yajurveda et les rasa à l'Ātharveda », Nātyaśāātra, I-17.

${ }^{33}$ Ānandavardhana, Dhvanyāloka, cité par Gnoli, op. cit. p. 61.

${ }^{34}$ Idem.

${ }^{35}$ saṃskāra
} 
l'esprit ${ }^{36}$ sans cesse sujet à modifications, ne s'applique pas àl'oubli qui conserve les souvenirs sous forme d'imprégnations inconscientes ${ }^{37}$, sources de servitude ${ }^{38}$.

Le pouvoir de la bhāvanā dans l'art réside en ce qu'elle transcende les limites des moyens de connaissance ordinaire, comme par exemple la mémoire, pour provoquer une jubilation délivrée des « cuirasses »,ce carcan des représentations communes. De même que la remémoration, l'expérience esthétique agit sur les imprégnations résiduelles inconscientes. En revanche, son mode d'action n'est pas la réitération des éléments psychiques, mais leur « universalisation». Extraites de leur réserve par le pouvoir de révélation, sollicitées par les facteurs artistiques à s'incarner, les dispositions d'esprit permanentes subissent un effet singulier, inattendu de sublimation dans la transcendance. La bhāvanā ne rend pas présent le passé, elle rend le présentéternel. Elle n'engage pas le concept mais l'affect. Elle n'est pas représentation, mais jubilation. Son pouvoir consiste à révéler le rasa, essence de la poésie ${ }^{39}$ et de l'art en général.

Mais qu'est-ce que le rasa? Interrogeons la définition nominale selon laquelle « le rasa est l'essence de l'art ».

Afin d'éclairer l'analyse logique, Abhinavagupta propose un examen chronologique, qui dissocie deux actes de perception ${ }^{40}$ ou d'expérience directe, bien que quasi-simultanés : la première perception est de l'ordre du sensible et de l'intellection. Par la lecture ou l'audition, le sens littéral des mots est compris (dénotation, connotation) alors même qu'ils sont vus (déchiffrement) ou entendus. Par les sons, les vibrations sont senties et leur source identifiée, par les mouvements et les gestes, les personnages sont vus et reconnus. Cette première perception des sens et de l'esprit, lorsqu'elle participe de tous les actes de l'existence ordinaire, en suppose une secondeparl'intérêt manifesté pour les objets de la perception et les représentations qui les accompagnent,et par une certaine implication dans l'action. Ainsi, la lecture des spéculations liturgiques qui mettent en action des personnages mythiques ou divins se présentent, en « seconde perception », comme une invitation, voire une injonction, à imiter leur exemple : l'énoncé «Sāmba a retrouvé la santé en priant le dieu Soleil » doit être interprété et universalisé par le dévot, éliminant son caractère particulier et circonstancié (circonscrit dans un temps, un espace, et un cadre culturel), en «tout homme priant le dieu Soleil retrouve sa santé » puis intégré en «je vais donc moi aussi prier pour me protéger des maladies »(Gnoli, p. 64). Dans l'art, de même, la première perception est immédiatement suivie d'une seconde, d'une tout autre nature (alaukika) : plus que d'universalisation, c'est un mouvement de transcendance ${ }^{41}$; ce qui est perçu ne vaut pas précisément pour tout lieu ou tout temps, mais hors de tout lieu et de tout temps. Ce qui se joue n'est pas un effort de la pensée, mais une expérience intuitive et spontanée de jubilation, au double sens du mot bhoga : aussi bien jouissance qu'expérience.

La bhāvanās'inscrit également dans le champ de la mystique. Elle est dans le shivaïsme tantrique l'actualisation de la pure énergie, un élan de l'imagination si fort et maîtrisé qu'il est capable d'arrêter tout mouvement de l'esprit.Celui-ci se stabilise alors sur l'énergie originelle manifestée en félicité, en résonance intérieure, en vibration sonore primordialeoù s'abîme le

\footnotetext{
${ }^{36}$ manas

${ }^{37}$ vāsanā

${ }^{38}$ Lire sur cette question Kapani (1992) pp. 285-298.

${ }^{39}$ kāvyārtha

${ }^{40}$ anubhāva

41 Les traductions littérales du terme sanskrit sādhāraṇī karana par «généralisation » ou encore « universalisation» (Bansat-Boudon, 1992, p. 152 ; Hulin, 1978, p. 355) ne me paraissent pas suffisamment rendre compte de ce passage à la dimension alaukika spécifique à l'art, et qui rapproche l'expérience esthétique de l'expérience mystique; je préfèrerais pour ma part le terme français de « sublimation ». Bansat-Boudon précise qu'il s'agit d'une « dépersonnalisation de l'émotion ».
} 
yogin, délivré de toute dualité, absorbé dans l'unité de la pure Conscience. Cette absorption elle-même exige néanmoins un effort soutenu pour faire échec aux impressions latentes, aux dispositions résiduelles dynamiques qui tendent à rétablir le sens de la dualité et l'idée de soi. La pratique consiste à renforcer les effets de la bhāvanā par la bhāvanā elle-même ; énergie créatrice de la conscience, elle est au-delà de la pensée dualiste conceptuelle, et en deçà de l'absorption parfaite. Elle s'exerce sans le secours de la pensée discursive, dans le silence d'un mouvement de conscience pré-langagière, intuitive et efficiente néanmoins, en deçà de la sublimation absolue. Elle définit, précise Lilian Silburn, «la zone spécifiquement mystique, zone obscure, silencieuse et calme de l'émergence, frange d'intuition qui borde la pensée où rien n'est encore cristallisé. Contemplation, ravissement, paix, parole, parole intérieure qui se cherche (...), élan de tout l'être, ces divers éléments se trouvent parfaitement fondus dans cette forme dynamique de la conscience, mais qui est trop simple et trop indéterminée pour faire l'objet d'une claire définition ${ }^{42}$. C'est peut-être pourquoi le concept de bhāvanā ne suffit pas à rendre compte des conditions de possibilité de la jouissance esthétique. Analysé dans sa glose du Nātyaśāstra par Abhinavagupta comme " pouvoir » des mots, ou de l'œuvre d'art en général, elle n'existe que sous l'impulsion de la force créatrice du poète ou de l'artiste d'une part, et de la sensibilité du contemplateur qui la perçoit, d'autre part, et dont rend compte, pour tenter de préciser l'effort de conceptualisation, le terme de pratibh $\bar{a}^{43}$.

\section{L’intuition créatrice}

Son sens, dans le domaine esthétique, est double: inspiration, pouvoir d'intuition, intuition créatrice, force créatrice selon le contexte en premier lieu ${ }^{44}$, et sensibilité, pouvoir d'intuition également, intuition contemplative, force ou pouvoir (sur soi-même) de contemplation par ailleurs ${ }^{45}$. Elle est une forme de perception directe, de caractère nondiscursif, qui désigne une fois encore chez Abhinavagupta l'intuition mystique aussi bien que l'intuition esthétique. Perception directe ${ }^{46}$, elle est l'œuvre de la conscience intuitive, révélation d'une vérité pré-discursive, à l'instar du troisième œil de Śiva qui perçoit toute forme d'existence présente, passée et future ; cette perception donne accès au réel, dégagé de ces obstacles à la connaissance que sont les catégories langagières, insinuées entre notre conscience et la réalité même. Peut-être Bergson s'y retrouverait-il, qui dénonce l'infirmité de la penséecommune par cette formulation bien connue : entre la nature et nous, entre nous et notre propre conscience, un voile s'interpose, voile épais pour le commun des hommes, voile léger, presque transparent, pour l'artiste et le poète. Vivre consiste à agir, ajoute-t-il, et vivre c'est n'accepter des objets que l'impression utile pour y répondre par des réactions appropriées ; les requis de l'existence pratique nous conduisent d'ordinaire à interposer nousmême ce voile, qui simplifie le réel en n'en laissant paraître que ce qui satisfait nos intérêts réduits par les représentations de nos désirs serviles, déterminés par les exigences de l'action. L'art écarte le voile des conventions sociales et des amenuisements discursifs, pour nous mettre face à face avec la réalité même. Le philosophe tantrique, affirmant que tout est conscience, oppose quant à lui deux attitudes exclusives : la pensée dualiste (nous dirions pensée d'entendement) qui implique la séparation artificielle du sujet et de l'objet, œuvre des

\footnotetext{
${ }^{42}$ Silburn, op. cit., p. 33.

${ }^{43}$ Le terme est polysémique, et peut signifier aussi bien le pouvoir de vision extatique, celui de comprendre sans effort et de communiquer avec les animaux, ou encore celui de la prémonition; dans ce cas, pratibhā est une forme de l'intelligence intuitive prajñā référée au futur, distincte de smrti, la mémoire ou conscience du passé, et mati conscience du présent.

${ }^{44}$ kārayitrī pratibhā, de l'ordre du savoir faire.

${ }^{45}$ bhāvayitrī pratibhā, de l'ordre du savoir être.

${ }^{46}$ sākșātkāra
} 
cuirasses ${ }^{47}$ transformant le libre infini en activités fragmentaires limitées par le temps, l'espace et la causalité, et la pensée de l'indifférenciation qui, par la prise de conscience et la vision intérieure, "perce l'écran » qui s'efface devant le «grand ciel lumineux », où brille toujours identique à elle-même la lumière indivise de la conscience absolue (éternelle vibration), laquelle a pour caractères essentiels la liberté ${ }^{48}$ et la béatitude.

Retenons simplement ici cette thèse métaphysique que la béatitude est l'essence de la Conscience, à laquelle accède le yogin (et le poète, l'artiste) par la pratique : il se libère ainsi progressivement des expériences accumulées, des sédiments et tendances inconscients, causes des cuirasses rigides et artificielles, de l'ignorance ${ }^{49}$ et de la souffrance ; la béatitude n'est pas un simple état affectif du sujet, dans la plénitude du désir sublimé : elle est ce dont tout procède, de sorte qu'à l'issue de sa quête d'absolu, "le yogin touche alors à la cime du bonheur et l'on peut dire que la félicité n'est pas en lui, mais que c'est lui qui repose en elle » (Silburn, p. 63). Mais que l'on ne s'y méprenne : certes, l'approche philosophique justifie l'appartenance de la pensée esthétique indienne à la métaphysique, et l'on ne comprendrait probablement rien à la théoriesans cela. Cette pensée n'en reste pas moins très phénoménologique dans son mode de construction au plus près de l'expérience (de l'artiste et du contemplateur).Si, par exemple, le concept de pratibha $\bar{a}$, intuition artistique, est conçu par la métaphysique comme une hypostase de l'intuition universelle de la Conscience en tantqu'émission créative (visarga), elle n'en est pas moins analyséecomme intuition esthétique vécue. L'analyse d'une œuvre tente toujours d'en cerner les modes d'organisation pratique et matérielle dont le sens (qui ne peut être que sens incarné incompatible avec l'idée d'une superposition duelle de la forme et du fond) est consubstantiel à l'expérience.

Il y est question d'un mouvement de forces en présence (un «pouvoir ») qui rend la conscience à elle-même, distanciée de son mode d'être servile, et auquel dès lors elle assiste comme spectatrice d'elle-même, et dont elle pourra dire, peut-être à la manière de Paul Valéry, c'était « je ne sais quel chant que je murmurais, ou plutôt qui se murmurait au moyen de moi $^{50} »$. Il y est question de l'énigme du surgissement de l'œuvre et de l'expérience ineffable qui l'accompagne. Ainsi faut-il comprendre pratibha $\bar{a}$, l'intuition esthétique : une force sublime qui, par la mise en œuvre maîtrisée de ce "pouvoir de révélation du rasa » qu'est la bhāvanā, installe la conscience dans une expérience singulière(conçue comme celle de sa nature propre), reposant en elle-même, dans la béatitude. La description de l'expérience et de ses conditions est si précise, qu'elle pourrait bien se passer de toute spéculation. Le lecteur moderne n'a pas de difficulté à distinguer la phénoménologie de l'expérience ellemême, telle que l'envisage Abhinavagupta, et son interprétation métaphysique. Rien n'empêche, à cet égard, de douter, comme le faisait Montaigne à propos des idées de Platon ou des atomes d'Épicure, que les philosophes indiens croyaient vraiment à leurs théories.Ils se méfiaient trop des constructions intellectuelles pour y adhérer tout à fait.

Quoi qu'il en soit, nous sommes loin du recours à l'irrationnel de la magie chère à l'ancienne tradition védique ; cette force sublime de la pratibhā n'est rien sinon le pouvoir de création de l'artiste qui produit l'œuvre, et par là seulement se révèle à lui-même : dans l'océan infini de la poésie, précise Ānandavardhana dans son Dhvanyāloka, le poète est le vrai et le seul créateur, et le tout grandit et se transforme comme il lui plaît de le créer. Il est vrai que l'acte de création est jubilatoire, il l'est même par essence, il n'est pas moins vrai qu'il s'effectue laborieusement, dans la solitude et l'inquiétude, le travail acharné à donner naissance et forme efficiente à la bhāvan $\bar{a}$, le doute et la concentration exclusive sur l'œuvre

\footnotetext{
${ }^{47}$ kañcuka

${ }^{48}$ svātantrya

${ }^{49}$ Śankkara, par exemple, appelle «surimposition » le fait de conférer au Soi des déterminations qui appartiennent en propre au corps, signe de l'ignorance métaphysique (Hulin, 1994).

${ }^{50}$ P. Valéry, « Poésie et pensée abstraite », Oeuvres, Gallimard, p. 1322.
} 
en train de se faire, harassé par la pensée de son travail comme le remarque Mānikyacandra dans son kāvyārthacintanapara (commentaire du Kāvyaprakāśa de Mammața). Cette dimension proprement humaine du travail dans toute sa matérialité a été évoquée tant et si bien que certains auteurs postérieurs à Abhinavagupta, comme Hemacandra, théoricien Jain $\mathrm{du} \mathrm{XII}^{\circ} \mathrm{s}$, ont résolu de distinguer deux temps dans la création, la production laborieuse de l'œuvre et sa contemplation sereine - afin, peut-être, de sauver maladroitement le paradoxe de la mise en présence de la douleur et de la joie dans l'acte créateur.

La même intuition est en œuvre chez le contemplateur, la puissance de contemplation (ou intuition contemplative). Elle suppose de la part du sujet une qualité nécessaire, sans quoi aucune saveur ne saurait être expérimentée, la sahrdayată, littéralement «le fait d'être pourvu d'un cœur» ou encore hrdayasamvāda «la correspondance des cœurs », la sympathie, la sensibilité esthétique. Abhinavagupta définit l'esthète comme toute personne dont le cœur possède un pouvoir d'intuition comme un miroir. La métaphore du miroir traduit cette idée que la même puissance de révélation du rasa est en acte dans la création et la contemplation ; la création est l'image originale, la condition de possibilité première de l'expérience esthétique est la création de l'œuvre elle-même puisque le poète, on l'a vu, est le seul créateur et que tout est comme il lui plaît de le faire. A l'identique de l'original, mais seulement perçu et non produit, comme son reflet dans le miroir, la contemplation manifeste le surgissement de la même puissance de révélation. La métaphore du miroir n'exprime pas seulement l'identité des forces en œuvre dans la création et la contemplation, mais également la nécessité d'un travail d'éducation; la possibilité de vibrer en sympathie avec l'artiste ou l'œuvre n'est pas spontanément donnée, et cette spontanéité de la révélation du rasa dont parlent les textes, qui distingue l'expérience esthétique de l'expérience mystique, beaucoup plus austère et laborieuse, suppose un pré-requis : l'apprentissage. L'apprentissage nécessite une fréquentation assidue des œuvres, un exercice répété de cette faculté qui, si elle est présente en tous, exige un travail susceptible de libérer sa puissance ; comme un miroir qu'on ne polirait pas suffisamment ou qu'on ne nettoierait jamais ne renverrait nul reflet, le « cœur » du sujet sans éducation ne saurait se laisser envahir par aucune vibration ${ }^{51}$, ni, par conséquent, éprouver la béatitude. Il faut, en somme, une pratique, une pratique de la joie si tant est que l'essence du rasa est la béatitude. Dans son Dhvanyālokalocana, Abhinavagupta affirme que « ceux qui ont la faculté de s'identifier à l'objet de la description, le miroir de leur cœur rendu limpide par l'étude et la pratique assidue de la poésie, ceux-là, dont le cœur répond [à celui du poète], ce sont les sahrdaya. Car il a été dit: la délectation de ce qui trouve le consentement du cœur révèle le rasa. Le corps en est envahi, comme le bois sec par le feu » (BansatBoudon, 1992,pp. 148-149; Gnoli, p. 65).

L'esprit parfois, mais le corps et surtout le «cœur », tels sont les lieux de l'expérience esthétique, étrangère à toute intellectualisation, qui procède de l'affect ; un affect que définit le repos de la conscience en elle-même. Le pouvoir de révélation du rasa dont l'œuvre est porteuse communique, comme par propagation de la vibration d'un corps à d'autres corps, la pratibhā de l'artiste à celui qui contemple, qui « entre en identité avec le cœur du poète » (Gnoli, p 65) ; ceci est rendu possible par la sympathie, l'affinité des dispositions mentales (bhāva), des imprégnations résiduelles et des dispositions acquises (vāsanāa, samskāara) qui déterminent les attentes et les préférences des esthètes. L'expérience puise ses ressources dans le psychisme inconscient, qui, du fait de la singularité de ses tendances, induit une sensibilité particulière qui incline à une dilection pour certains artistes ou certaines œuvres plutôt que d'autres. Le sujet d'une nature aimable éprouve un penchant plus affirmé pour les affects d'amour, celui d'une nature courageuse pour ceux d'héroïsme. Ainsi, l'éducation consiste non seulement à développer la sensibilité esthétique sans quoi aucune expérience n'est possible,

\footnotetext{
${ }^{51}$ spanda
} 
mais aussi, et peut-être surtout, la singularité de cette sensibilité. La catégorie des guna (en tant que ceux-ci affectent nos dispositions et structurent notre identité -svabhāva) étaye l'effort de théorisation des enjeux pour une telle éducation: dissiper le principe obscur et ténébreux de la nescience (tamas) et libérer la luminosité sereine et pénétrante de la conscience (sattva) tout en disciplinant l'énergie active dans ses excès passionnés (rajas). Voici un exemple, appliqué au yoga, du caractère heuristique de la catégorie de guna (Kapani, 1993, p. 421):

«Le sattva a pour forme la clarté, mélangé avec le rajas et le tamas il se plaît dans le pouvoir et les objets des sens. Alors que ce même sattva, lorsqu'il est pénétré par le tamas, tend vers le vice, l'ignorance, le non-détachement et le non-pouvoir. Et ce même sattva, lorsque le voile de l'égarement a disparu, illuminé alors de toute part, mais encore pénétré d'une certaine quantité de rajas, tend vers la vertu, la connaissance, le détachement et le pouvoir. Le même sattva, une fois libéré des particules souillées provenant du rajas, établi dans sa propre nature, (...) tend vers la mise en position stable du psychisme (...) », où l'Énergie de la Conscience « est pure et infinie ».

Affectées de sattva dans l'expérience esthétique, nos dispositions permettent l'accès à la joie. Telle est donc la visée ultime de l'éducation esthétique, et en même temps son fondement comme en témoigne cette première strophe propitiatoire qu'Abhinavagupta inscrit en exergue de son Abhinavabhāratī: «De ceux qui s'identifient (au spectacle) par la correspondance des cours, Il [Siva] obtient (...) qu'aussitôt ils s'enchantent de la transformation suprême où l'on est joie vibrante » (Bansat-Boudon, 1992, p. 151).

\section{Communion esthétique et intuition directe}

Sympathie, identité, unisson sont les liens qui signalent la théorisation de ce qui est en œuvre dans l'expérience de l'artiste et du contemplateur. Nous pourrions préciser des contemplateurs. La sensibilité esthétique qui détermine l'intuition contemplative trouve en effet une forme inédite d'épanouissement dans la communion des consciences, qui participe de la métamorphose. Cette communion est rendue doublement possible par les impressions latentes qui structurent la formation du psychisme, et par la nature même de la délectation qui transcende les individualités. La multiplicité indéfinie des vies antérieures a en effet imprégné le linga d'une variété abondante de traces latentes qui constituent une réserve potentielle, commune à toutes les consciences malgré la spécificité de leurs penchants particuliers, et rendent possible le partage des affections. Le courageux a connu la peur, le détaché a connu la fureur, le sage a un jour rencontré l'amour ce qui, d'ailleurs, est la cause de la menace perpétuelle qui le guette de voir un jour réduites à néant des années d'ascèse par la beauté des femmes. De plus, l'expérience esthétique s'épanouit dans un mouvement de transcendance éliminant les particularités individuelles d'ordinaire circonscrites par des limitations de temps, d'espace, et sensibles à la causalité chaotique des phénomènes extérieurs et internes, qui déterminent les représentations mentales et les passions. Cette sublimation de la perception qui élimine le sentiment de l'individualité des sujets, les rassemble du même coup dans un sentiment d'unité, celui de former une seule même conscience en état de délectation. Abhinavagupta précise même que plus le nombre de contemplateurs croît, plus la délectation s'intensifie, comme si la multiplicité des miroirs du cœur se reflétant mutuellement les uns les autres rendait plus présente, plus réelle, plus efficiente l'image originale qu'ils accueillent. L'état d'identité des consciences unies dans l'acte de perception esthétique amplifie à l'extrême la jubilation. La conscience, explique Abhinavagupta dans son Tantrāloka, atteint en ces circonstances de communion «un état d'unité, et entre ainsi dans une béatitude faite de plénitude et de perfection. En vertu de l'absence de toute cause de contraction telles l'envie, la jalousie etc., la conscience se découvre en mouvement d'expansion, libre de tout 
obstacle, et envahie de béatitude » (Gnoli, p. 70). Sous l'angle métaphysique, cette contemplation est décrite comme une expérience inouïe de la vraie nature du Soi, Conscience universelle également présente en toutes ses manifestations particulières qui s'ignorent comme telles dans l'illusion de l'existence ordinaire, et découvrent alors leur identité réelle et absolue, dans l'indifférenciation de la communion esthétique. La puissance créatrice et contemplative de la pratibhā, portée par la sensibilité des sujets et amplifiée par leur communion dans la délectation est prodigieuse, et se remarque non seulement par son « succès humain ${ }^{52}$ » en quoi consistent toutes ces manifestations vocales et corporelles que sont les rires, les exclamations ou encore la chair de poule, les acclamations et gestes d'enthousiasme, mais aussi par son «succès divin ${ }^{53}$ » qui saisit un auditoire entier, captivé dans un silence troublant. Sans doute ce dernier est-il le plus souhaitable, s'agissant de la béatitude. L'exubérance joyeuse certes réconcilie le sujet avec le monde, qui goûte l'évanouissement de tout ressentiment, mais l'intériorité sereine le réconcilie avec lui-même, alors qu'il découvre une possible perfection dans la finitude. La référence d'inspiration védique au pouvoir magique de l'œuvre, pour expliquer ce passage si mystérieusement soudain à une jubilation intense et prodigieuse, est bien plus d'ordre généalogique que philosophique.

L'enjeu, par quoi l'on juge la valeur de l'art, se formulerait schématiquement comme suit : la création et la contemplation de telle œuvre maintiennent-elles la conscience dans l'ignorance et la douleur, le sentiment de l'individuation et le sens d'une existence séparée entretenus par une agitation mentale déterminée du plus profond du psychisme, ou bien l'affranchissent-elles de ses limites pour la faire accéder à la connaissance de sa vraie nature, dans l'expérience de l'unité et de la béatitude ? Cette dernière visée nous aide à mieux saisir la spécificité de l'esthétique indienne : l'œuvre n'y est pas pensée pour elle-même, mais comme médiation; sa production n'est pas la finalité de l'art, dont le maître mot n'est pas tant la création que la pratique; plus qu'il ne présente une œuvre, l'artiste témoigne et invite : il témoigne de la perfection dont l'homme est capable - entendons son propre effort de délivrance et sa puissance de béatitude - et invite à participer de cet effort, à l'engager pour soi-même.

La pratique est une exigence qui s'impose à chacun, pour peu que l'on soit « l'ami de la sagesse » dirions-nous. De sorte que ce qui donne son sens à l'art n'est pas la présentation d'une œuvre géniale, exemplaire et achevée, mais le travail requis pour sa réalisation : plutôt que l'œuvre, la pratique, plutôt que la représentation ou l'exposition, l'enseignement. Où l'on retrouve l'importance considérable, pour ne pas dire la valeur essentielle, de la relation de maître à disciple (Bugault, 1994, pp. 75-89; Siauve 1980) : ce qui explique l'oralité de la tradition, ce n'est pas l'ignorance de l'écriture, ni un quelconque archaïsme pré-conceptuel, mais l'enjeu délibéré de l'art comme exercice spirituel, comme "conversion de l'âme », en termes métaphysiques indiens commeeffort de délivrance, d'identification à l'absolu. Le maître et le disciple, mais également l'artiste et le sahrdaya, sont mis en présence, par la médiation de l'art, d'une possible réalisation de l'infini en eux, qui s'éprouve sur le mode de la béatitude, ānanda, ou l'affect de joie au comble de sa puissance. Il n'est alors question que de pratique : la maîtrise des facteurs artistiques, auxquels l'exercice du « pouvoir » d'intuition donne toute leur efficience, est exigée par notre corporéité (la philosophie elle-même soumet pour une large part ses investigations aux pratiques corporelles des postures et du souffle). Ce pouvoir d'intuition est aussi bien "pouvoir de béatitude » et l'on pourrait ainsi résumer : la création est puissance de joie et la joie est puissance de conversion.

\footnotetext{
${ }^{52}$ mānușī siddhi

${ }^{53}$ daivi siddhi
} 
Outre les conditions de possibilité de l'expérience esthétique, comment comprendre la nature même de cette expérience ? "Quelle est donc, s'est interrogé Abhinavagupta, la vraie nature du rasa?».

L'expérience esthétique est dite $d$ ' « intuition directe ${ }^{54}$. Cette intuition consiste en une forme de cognition, consistant en l'acte de savourer, caractérisée par la sublimation des objets de la perception, ainsi que par l'absence d'obstacles ${ }^{55}$. La formule paraît sibylline : le terme d'intuition suffit à l'éclairer ; il exprime, on le sait, une modalité de la connaissance qui, sans médiation, met l'esprit en présence de son objet. Nous pouvons l'appréhender au sens où Bergson la distingue de l'intelligence, qui a pour vocation de penser la matière, et la définit comme la seule manière de connaître véritablement, directement, l'esprit, et d'atteindre l'absolu en coïncidant avec ce que l'objet a d'unique et d'inexprimable. Dans un contexte théorique différent mais avec un certain air de famille, la médiation de l'intellect et de ses représentations est, selon Abhinavagupta, totalement écartée de l'intuition esthétique qui, débarrassée de ces «obstacles », accède directement à son objet, la délectation pure de la conscience par elle-même. Les objets de la perception ont été universalisés, transcendés dans un même mouvement, de sorte que ce que la conscience savoure n'est rien d'autre que sa propre nature (la béatitude) mise en présence, révélée à elle-même par la puissance créatrice (le «pouvoir» de création ou de contemplation). La perception ou intuition directe est de l'ordre de la connaissance, puisqu'elle rend possible le dépassement des causes d'ignorance (les obstacles), les fluctuations de la pensée dualiste, l'agitation mentale, les représentations égotiques, et qu'elle conduit la conscience à la contemplation d'elle-même.

La transcendance caractérise rigoureusement l'intuition directe dans l'expérience esthétique. Dans la dramaturgie par exemple, elle se définit par ce que l'on pourrait nommer la distanciation. Les deux réalités de l'acteur et du personnage représentés sont niées: l'acteur ne se présente pas sur scène comme tel, et c'est bien le rôle des costumes, du maquillage, des masques, des transformations de voix, des décors etc. que d'éliminer toute référence à un personnage réel comme le sont ceux que l'on rencontre d'ordinaire dans la rue ; à l'instar des fêtes religieuses où les danseurs sont masqués, leur corps peint et orné pour leur conférer un caractère sacré, les acteurs sont métamorphosés de sorte qu'ils relèvent désormais du supra-mondain (alaukika). Mais le personnage n'est pas non plus imaginé vrai, et cela distingue le théâtre du rituel magique religieux où les danseurs sont investis de la présence des dieux ou des esprits, comme dans les rites de possession. Le personnage joué n'est réellement présent sur scène pour personne, sinon pour un petit enfant ou un halluciné ; chacun sait qu'il n'est qu'un vibhāva, et l'effet d'idéalisation en est renforcé. Ni véritablement acteur (on l'oublie) ni véritablement personnage (on n'y adhère que partiellement), celui qui se meut sur scène se prête doublement à la distanciation, universalisant l'espace et le temps ; les évènements ${ }^{56}$ et les sentiments ${ }^{57}$ ne sont pas réels, les mimiques $^{58}$ et les manifestations corporelles des émotions ${ }^{59}$ sont fictives, qui autorisent aussi bien une "généralisation» des rapports de causalité. En même temps, les spectateurs ne restent pas indifférents, et se trouvent emportés, pour peu qu'ils aient acquis « la limpidité du cœur $^{60} \gg$, par la bhāvanā, le pouvoir de révélation des affects. Ainsi, la conscience se dégage totalement de toute préoccupation, de toute idée même, du monde ordinaire, de tout intérêt fini, bref, de tout jugement.

\footnotetext{
${ }^{54}$ sākșātkāra

${ }^{55}$ nirvighna

${ }^{56}$ vibhāva

${ }^{57}$ vyabhicāribhāva

${ }^{58}$ anubhāva

${ }^{59}$ sattvikabhāva

${ }^{60}$ hrdayavaimalya
} 
Le langage poétique y trouve tout son sens : là où le langage ordinaire ou prosaïque inscrit la conscience dans le monde des réalités sensibles et immédiates, l'assujettit à l'action que commandent les représentations utiles, les informations, les descriptions de fait, renforce le sentiment de l'individualité et l'identification au moi empirique, traduit fidèlement les injonctions inconscientes des impressions résiduelles dynamiques qui nous déterminent, le langage poétique nous en affranchit, en vertu du pouvoir de révélation du rasa propre aux mots. Distanciation et absence de jugement sont les caractéristiques de l'intuition directe qui définit l'expérience esthétique.

Le qualificatif «directe » est nécessité non pas pour définir l'essence de l'intuition ce qui serait tautologique, mais en écho à la notion d' «obstacle ${ }^{61} »$, qui entre dans l'analyse du concept d'intuition esthétique. Cette notion désigne, en effet, tous les éléments qui contrarient la possibilité de l'expérience esthétique (les théoriciens se sont efforcés d'en dresser la liste et d'en justifier les effets), ou dont à l'inverse la résistance est vaincue par la puissance créatrice. La transcendance consiste précisément en ce mouvement intuitif de la conscience qui dissipe la prégnance des obstacles. Leur multiplicité envahit le processus de perception ordinaire de la conscience, qui les subit comme autant de déterminations contraignantes capables de le maintenir dans l'ignorance et l'illusion, causes de la douleur et de la servitude. Les interactions répétées et imprévisibles des multiples obstacles entraînent le mouvement indéfini des fluctuations mentales qui fragmentent l'activité de la conscience, perpétuellement arrachée à elle-même. De même que le yoga vise l'arrêt des citta-vrtti, l'expérience esthétique implique la suppression des obstacles, qui établit la conscience dans l'unicité, la densité d'une concentration exclusive ${ }^{62}$. L'esprit en état de savourer est tout entier absorbé dans l'objet de sa délectation, à l'exclusion de tout autre chose, libre de toute perception sensorielle, de toute représentation mentale, de toute distinction de soi et d'autrui, en une présence sublimée dans le sentiment d'éternité, dégagée du système ordinaire de coordonnées spatiales : il se tient en repos dans l'affect. L'un des exemples proposés par Abhinavagupta pour illustrer cela, désormais fameux ${ }^{63}$, est extrait d'une scène de l'œuvre de Kālidāsa, Śakuntalā(I-2), qui présente dans un tableau de chasse la poursuite par le roi Dusyanta d'une gazelle ${ }^{64}$ : la situation dramatique créée par le texte, la musique et le jeu d'acteurs provoque la sublimation de la disposition d'esprit (sthāyibhāva) de la peur ${ }^{65}$ en la saveur esthétique (rasa) du Terrible $^{66}$. Son commentaire du poème, présenté ici dans la traduction de Michel Hulin (1978), insiste sur l'élimination des obstacles qu'opère la perception intuitive directe dans l'expérience esthétique :

«Après la saisie du sens (littéral) de telles phrases se produit immédiatement une perception mentale, intuitive, dans laquelle se trouvent éliminées les perceptions de temps, etc. contenues dans ces phrases. Dans une telle perception, le jeune daim, par exemple, apparaît dépourvu de toute particularité et (l'acteur le représentant) qui communique l'effroi (aux spectateurs) n'est pas absolument réel. (Se manifeste alors) la peur en soi, une peur que ne délimitent plus le temps ni l'espace. Elle diffère des impressions ordinaires de peur, du genre : «j'ai peur » ou «mon ennemi, mon ami, un tel a peur », car ces dernières abondent en obstacles (à la jouissance esthétique), du fait qu'elles comportent nécessairement l'apparition de nouvelles réactions d'évitement, etc. de caractère (tantôt) agréable, (tantôt)

\footnotetext{
${ }^{61}$ vighna

${ }^{62}$ ekāgra

${ }^{63}$ Cf. Gnoli p. 67 ; Hulin, 1978, p. 349 ; Bansat-Boudon, 1992, p. 147 ; Poggi, p. 137.

${ }^{64}$ «(Le daim) ployant gracieusement son cou, jette de temps à autre un regard vers le char qui le poursuit. Son arrière-train ne cesse de se mouvoir vers l'avant, comme s'il craignait la descente de la flèche. Il jonche le chemin d'herbes à demi mâchées et de gouttelettes tombant de sa gueule béante d'épuisement. Oh ! ses bonds puissants font qu'il se meut surtout dans les airs et fort peu sur le sol !», trad. M. Hulin, op. cit., p. 348.

${ }^{65}$ bhaya

${ }^{66}$ bhayānaka
} 
pénible. Au contraire, cette perception ne comporte pas d'obstacles et pénètre directement dans le cœur. C'est le rasa du Terrible qui danse, pour ainsi dire, devant nos yeux ».

Les obstacles sont en somme tout ce qui participe d'un acte de perception ordinaire (laukika) tant en regard de l'objet perçu (les particularités -viśeșa-des personnages, comme le daim, et des acteurs) que des conditions de la perception (l'espace-temps spécifiques à l'œuvre et sa présentation) ou du sujet percevant (les émotions et sentiments du quotidien vécu) et qui suscitent spontanément des comportements d'action et de réaction liés à la protection de soi (la peur, en l'occurrence, provoque la fuite ou l'agressivité) comme aux représentations empiriques et pragmatiques de participation personnelle aux évènements. Ils sont source de «contraction» de la conscience sur elle-même qui s'identifie à son individualité subjective et séparée; éliminés par la puissance de l'intuition directe, la conscience est rendue à son mouvement essentiel d' "expansion », prolongé par la réflexion des autres consciences en communion - rendue possible par le sensus communis des impressions latentes. Ce mouvement accompagne l'état de «densité » (ekaghanatā) de la conscience, qui indique son immunité conquise contre la perturbation des obstacles à la délectation. Ainsi l'obstacle n'est pas simplement ce qui participe de la perception ordinaire, mais plus précisément ce qui contrarie et entrave l'expérience du «supra-mondain» (alaukika), l'extra-ordinaire dans l'art. Les diverses disciplines enseignent l'art de lever les obstacles, dont Abhinavagupta dresse un tableau taxinomique pour l'art dramatique ${ }^{67}$; «Les éléments qui éliminent les obstacles à la perception esthétique sont les Déterminants, etc. », affirme-t-il.

La transcendance manifeste cette capacité dont la conscience fait preuve de tendre vers un au-delà d'elle-même lorsque, d'ordinaire circonscrite dans une appréhension du monde limitée par l'espace et le temps, elle accède soudain, dégagée de tout obstacle, à un sentiment d'universalité et à l'expérience de l'unité : l'éternité au cœur du présent. Cette expérience a pour seul contenu la jouissance de l'affect, la délectation, et se définit comme le repos de la conscience en elle-même, savourant sa propre béatitude. L'essence du rasa est dès lors, comme en dernière analyse, la béatitude.

\section{La jubilation}

Essence du rasa, la béatitude l'est aussi bien de la conscience elle-même ; ce que la conscience savoure dans l'expérience esthétique, c'est donc sa propre essence. L'idée paradoxale d'un repos en mouvement (repos entendu comme quiétude ineffable) est chère à la métaphysique cachemirienne qui conçoit l'Absolu comme vibration permanente originelle, et l'être comme une dynamique d'absolue liberté ${ }^{68}$. Dans l'œuvre, le repos inclut le mouvement, ou plutôt le mouvement «s'universalise » en délectation dans le repos où il se tient, rendu à

\footnotetext{
${ }^{67}$ Les obstacles sont au nombre de sept : $1 /$ impossibilité de la perception pour cause d'artifice ; $2 /$ perception des déterminations spatio-temporelles; $3 /$ perception de sensations étrangères à l'expérience esthétique ; 4/ infirmité des moyens de perception ; $5 /$ manque de plausibilité ou de vraisemblance ; 6/ confusion des affects $7 /$ possibilité du doute ; Abhinavagupta les analyse et évoque les techniques de résolution propres à chacun (Gnoli, pp. 77 sq.).

${ }^{68}$ Que l'on songe, de façon contrastée, à l'origine de l'œuvre d'art selon Heidegger : «Mettre en place un monde et faire venir la terre sont deux traits essentiels dans l'être-œuvre. Ils s'appartiennent l'un l'autre dans l'unité de l'être-œuvre. Nous recherchons cette unité lorsque nous pensons l'immanence de l'œuvre et tentons de dire le calme contenu et le recueil de reposer sur soi.

Mais, à supposer qu'il y ait là quelque chose de fondé, ne venons-nous pas, avec ces traits essentiels, de caractériser dans l'œuvre non pas un repos, mais bien plutôt un avènement? Qu'est-ce en effet que le repos, sinon le contraire du mouvement? Bien sûr. Mais il n'est pas un contraire qui exclut le mouvement : il l'inclut » (Heidegger, Chemins qui ne mènent nulle part, Gallimard, 1992, p. 51-52).
} 
sa nature de vibration originelle. La signification de l'art se range sous les catégories de «pouvoir », de puissance, catégories de la transformation ou du passage, qui signalent sa faculté métamorphique, pour utiliser une notion géologique. Le terme de repos ne se réduit donc pas à son acception première, celle de rémission des agitations (encore qu'elle soit tout à fait importante), il désigne l'essence même de l'expérience esthétique, la béatitude. A l'expérience esthétique correspond une conscience apaisée, délivrée des fluctuations et des agitations de la pensée: elle est dite en repos parce que toute entière offerte à la contemplation d'elle-même, et fermement assurée contre quelque mouvement perturbateur que ce soit de l'esprit. Elle se trouve également saturée d'une jubilation ineffable, dont Bhața Nāyaka affirme qu'elle est « du même ordre que la jouissance du suprême brahman » (Gnoli, p. 56). Cette expérience de jubilation est dite camatkāra ${ }^{69}$, terme récurrent dans les textes sur la théorie esthétique et les spéculations métaphysiques, et qui porte toujours l'idée d'un saisissement en présence d'un événement qui envahit soudain tout le champ de la conscience.

Le poéticien Viśvanātha, dans son Sāhityadarpaṇa, le présente comme exprimant l'état de «conscience en expansion » (cittavistāra). De manière générale il qualifie en esthétique la teneur particulière de la jubilation dans l'art : camatkāra désigne l'immersion de la conscience dans la délectation de l'œuvre. Abhinavagupta le définit précisément ainsi : «état d'immersion totale en une jubilation caractérisée par un sentiment de plénitude intérieure » (Gnoli, p. 74). On peut le traduire par le terme d'émerveillement si l'on retient le caractère inouï et prodigieux (alaukika) de la jubilation, ou par celui de ravissement si l'on insiste sur son mouvement de transcendance, qui arrache la conscience aux déterminations de sa subjectivité ; le mot englobe à la fois le sens d'un enlèvement sous l'effet d'une force et celui de porter à un état de joie suprême. Le concept de camatkāra désigne le mouvement psychique de manifestation de la béatitude, elle-même expression de l'absolu. Que ce soit par la médiation des pratiques mystiques ou artistiques, camatkāra est toujours l'avènement au sein de la conscience individuelle de la béatitude, qui transcende ses propres limitations par la dissipation des obstacles à la connaissance (la délectation est une connaissance intuitive par la conscience de sa propre nature). L'Agnipurāna(339-1-2) emploie pour décrire l'expérience de la béatitude une terminologie commune aux domaines esthétique et spirituel :

«Il est dit dans le Vedānta que le brahman est immuable, suprême, éternel, absolu, omniprésent et unique ; il est conscience, lumière et Seigneur. Lorsque la béatitude essentielle à ce principe (de brahman) se manifeste, cette manifestation est ce que l'on nomme Conscience, camatkāra ou rasa ».

On mesure bien la distance qui sépare la joie ainsi comprise de toute référence au sentiment. La conscience, parce qu'elle est dégagée de tout « obstacle », ne subit plus rien du cycle circonstanciel des affections hétéronomes, qui alternent perpétuellement entre joie et tristesse. Elle les transcende, de même que tout discours, pour s'immerger dans une activité intuitive $\mathrm{e}^{70}$ qui consiste, dans le procès de création et de contemplation artistiques, à savourer la découverte assurée de sa propre nature essentielle, la béatitude ${ }^{71}$, en une jubilation ${ }^{72}$ illimitée et sans obstacle ${ }^{73}$. La capacité à s'émerveiller, qui doit faire l'objet d'une éducation et d'un travail sur soi, se présente comme l'antinomie de l'inertie, source d'ignorance ; aussi bien en est-elle l'antidote, en tant que puissance de joie (Hulin, 1978, p. 327) :

\footnotetext{
${ }^{69}$ L'étymologie fait dériver le mot de l'interjection camat qui exprime une surprise, associée à kāra (de la racine verbale $\left.k s_{-}^{-}\right)$qui signifie l'acte de prononcer une telle interjection. Abhinavagupta accepte une seconde interprétation selon laquelle camat est dérivé de cam qui signifie déguster, dont il est le participe présent. Ceci accentue la référence au champ sémantique de rasa, rasana, la saveur, l'acte de savourer.

${ }^{70}$ sākșātkāra

${ }^{71} \bar{a}$ nanda

${ }^{72}$ camatkāra

${ }^{73}$ nirvighna
} 
«L'inertie n'est pas autre chose qu'une totale absence d'émerveillement et (inversement) ce qu'on appelle «sensibilité » - sahrrdayatā - ne consiste qu'en une immersion dans un intense émerveillement où l'énergie virile est en état de tumulte».

Cette capacité n'est pas donnée, et "seuls ceux dont les cœurs sont nourris par cette énergie, engagés dans la constante pratique de la joie [d'ordre mystique ou esthétique] connaissent un intense émerveillement» (Gnoli, p. 73). L'éducation ne porte pas uniquement sur la fréquentation assidue des œuvres, qui développe la sensibilité en polissant «le miroir du cœur » comme je l'ai suggéré plus haut, mais également - essentiellement - sur le rapport de la conscience à elle-même, à sa propre nature béatifique; la «constante pratique de la joie », outre la jubilation esthétique, découvre à l'homme ses potentialités spirituelles ; ce que l'activité discursive de la pensée ne lui permet pas même d'entrevoir, il l'expérimente dans la perception intuitive directe, affranchi de tout obstacle à la connaissance. L'expression de F. Alquié rapportée à la vérité dans l'art de «savoir affectif», si l'on entend par affectif « de l'affect de joie », fait joliment écho à cette thèse.

La préoccupation de la joie, dès lors qu'elle n'est plus la simple poursuite des désirs vains, ni le signe incertain de l'espérance, non plus que l'affection circonstancielle née d'une détermination extérieure, mais le dynamisme de l'existence tout entière - comme «essence de la conscience universelle» selon la métaphysique tantrique - mérite une attention interrogatrice et active. L'expérience esthétique du ravissement est d'autant plus puissante que ses effets, ses impressions dans le psychisme, perdurent longtemps après que la contemplation de l'œuvre eût pris fin. Le rasa révélé dans la rencontre de la forme de l'œuvre (les «facteurs artistiques ») et de l'intuition créatrice se caractérise par un émerveillement capable de perdurer, à proportion de la sensibilité esthétique ; la conscience, envahie «par l'émerveillement, continue au moyen de son propre soi (svātmarūpa) à tout percevoir sous cette lumière »(Gnoli, p.113). La joie essentielle du ravissement, loin du simple souvenir d'un plaisir achevé, accompagne l'existence, et dure bien plus que les quelques jours de la représentation théâtrale indienne, comme on l'a parfois compris. Le rasa, qui fait affleurer à la conscience les impressions et imprégnations résiduelles, qui sollicite les dispositions acquises et les tendances héritées du passé, n'a rien de la mémoire (smrti) ni des modes opératoires ordinaires de l'esprit. Les couches subconscientes du psychisme, en l'état des sthāyibhāva, les dispositions d'esprit permanentes, sont requises dans un mouvement qui en modifie la nature, et infléchit leur activité dynamique, en stérilisant leurs germes au feu de la béatitude. Abhinavagupta affirme sans ambiguïté la puissance de l'émerveillement : «En vertu de la perception du rasa, (...) cette forme de conscience reste profondément fixée dans le cour, comme une flèche, d'une façon telle qu'elle ne peut par aucun effort être possiblement négligée, ni seulement extraite » (idem). Les dépôts lumineux, sattviques, des rasa au plus intime du psychisme, participent de l'effort de délivrance, de conversion de l'âme. Plus encore, il n'est que d'entretenir la présence de camatkāra au cœur de la conscience par une activité permanente du pouvoir d'intuition, pour accroître cet effort. Il n'est que de pratiquer la joie.

Cette jubilation caractérisée par un sentiment de plénitude intérieure qu'est camatkāra désigne la nature de l'expérience esthétique ; il faut en conclure que le rasa a pour essence la béatitude, ce que confirme Abhinavagupta : «Tous les rasa consistent donc en béatitude » (ibid., p. 90). La délectation dans l'art, que l'absence de désirs et d'idées (d'obstacles) rend possible, consiste en un acte de savourer les dispositions d'esprit permanentes, les affects latents, réactivés et manifestés en affects esthétiques ou rasa. Le rasa est ce qui est susceptible d'être savouré, telle est la définition du Nātyaśāstra; il est en quelque chose la manifestation 
épanouie des dispositions latentes; celles-ci demeurent inaccessibles au plus obscur de l'inconscient, et déterminent, dans la vie ordinaire, les affections qui nous asservissent et nous attachent à la douleur qui habite l'enchaînement irrationnel et chaotique des émotions, des sentiments et des désirs. Telles, elles ne sauraient faire l'objet d'aucune délectation; sollicitées par les causes et effets de l'existence commune, elles restent le plus souvent source de servitude, les affections fussent-elles de joies et de plaisirs passagers. Les activités «mondaines » engendrent, outre des discours, des affections au caractère servile nécessairement frappées d'incomplétude ; «comme l'a dit le maître Patañjali : "Le fait que Caitra soit épris d'une femme ne signifie pas qu'il se désintéresse des autres » (Yogasütra, Vyāsabhāssya, II 4). Dans la mesure donc où il entre en rapport avec ces (nouveaux désirs), son plaisir est incomplet et ne représente pas la suprême félicité. Et tel est le cas de toute jouissance mondaine. Ne dit-on pas : «Dans la possession on craint la séparation à venir » et : "Un objet (possédé) en fait désirer un autre, comment pourrait-il procurer un (réel) plaisir? ». Parce qu'elle ne peut rompre complètement avec la recherche de (biens) distincts (du Soi), elle demeure incomplète »(Hulin, 1978, p. 324 ; Gnoli, pp. 88-89.

La pratique des yogin, à l'inverse, les délivre non seulement de tout discours, mais aussi de toute tonalité affective, puisque leur effort tend vers la destruction de toute efficience des samskāra (donc des sthāyibhāva), sinon des sampskāra eux-mêmes : le dispositif inconscient des samskāra d'arrêt ou d'inhibition(nirodha-samnskāra) qu'élabore le yoga prive ceux-ci de tout contenu empirique ou sapientiel, provoquant l'arrêt de toute modification de l'esprit.

En conséquence, il apparaît que seule l'intuition esthétique est capable d'épanouir les affects dans la béatitude d'une conscience en délectation en ce que, sollicités dans la rencontre des puissances créative et contemplativepar la médiation de l'œuvre, ils subissent un mouvement de sublimation qui les débarrasse de tout penchant au particularisme, de tout lien avec une quelconque représentation de désir ou d'intérêt, de toute anxiété comme de toute espérance. On peut ainsi à bon droit parler d'épanouissement de l'affect dans l'art. Quoi d'autre que l'œuvre, demande à peu près Abhinavagupta, possède la faculté de révéler le rasa?

Le rasa n'est pas «quelque chose qui soit déjà réalisé » mais le privilège de l'art, l'apanage de l'œuvre et la prérogative de son pouvoir de révélation. Il dure autant que dure la délectation, et disparaît avec elle, insiste Abhinavagupta. Il suggère par là non pas l'idée chronologique d'un évanouissement spontané et immédiat du rasa à l'instant précis où cesse la présentation de l'œuvre - j'ai au contraire évoqué sa force de jubilation par delà ce moment - mais celle, logique, de leur corrélat obligé. Le rasa est le domaine propre de l'art, et son essence est de béatitude. Ce second point présente un paradoxe : si l'essence du rasa est la béatitude, et que, comme Abhinavagupta l'écrit lui-même tous les rasa consistent en béatitude, comment se peut-il qu'il y ait expérience du Furieux, du Pathétique, du Terrible ou même de l'Odieux? S'ils s'éprouvent en tant que «félicité de la lumière indivise prenant la forme d'une délectation de notre propre conscience », si «ce qui est goûté dans l'expérience esthétique, c'est notre propre conscience saturée de félicité », comment justifier que puisse danser devant nos yeux le rasa du Terrible, cette manifestation de «la peur en soi », ou encore le dégoût en soi ?

Si le contemplateur n'est ni indifférent, comme le yogin dans sa pratique, ni animé par un dégoût ou une peur pour lui-même comme c'est le cas dans la vie ordinaire, et si enfin tous les rasa sont constitués de béatitude, pourquoi les distinguer et pourquoi surtout ces affects de «tristesse », comme dirait Spinoza ? On peut s'accorder sur l'idée de trouver plaisir à jouer comme l'enfant, en toute sécurité, à «fais-moi peur », mais n'y a-t-il pas contradiction à assimiler en une même expérience fureur et félicité ? Et par ailleurs, un seul rasa, la béatitude ou la sérénité en soi, ne suffit-il pas à l'art? 
C'est que, précisément, l'art se distingue des disciplines austères de la mystique : chacun ne peut devenir yogin que pour lui-même, et hormis la relation d'enseignement, doit pratiquer «à l'intérieur d'une petite cellule de la dimension d'un arc, exempte de pierres, de feu et d'eau, et située dans un endroit solitaire ${ }^{74}$. La méditation est une discipline de sévérité, de renoncement et de solitude. L'art en revanche - hormis le temps d'intense apprentissage $(s \bar{a}$ dhanāa qui impose éventuellement la même austérité - s'offre à la contemplation et se partage, pour peu que l'on dispose d'une sensibilité suffisante. L'art lie l'expérience de la joie au sensus communis des affects que partagent tous les hommes, qui les relient entre eux dans leur existence, et aux contenus psychiques subjectifs qui colorent leur vie. L'art, certes, transcende, mais en célébrant; à l'injonction nietzschéenne «il faut spiritualiser les passions », il répond éminemment, et résolument. En plongeant au plus intime et au plus secret de la conscience, il crée des affects qui la métamorphosent; alchimie singulière, il convertit la peur, l'horreur, la tristesse ou l'aversion dont il nourrit ses œuvres, en paix -śánta - et en jubilation. L'art est le prédateur de la souffrance, du vice, du ressentiment et de la haine : il y trouve sa substance, qu'il convertit en félicité. L'art célèbre le tout de la vie, y compris sa douleur; non point parce qu'il l'imite, en une sorte de catharsis à courte distance d'une obscure fascination pour son objet, mais parce qu'il en dévoile le recel. L'art creuse au cœur même de la finitude pour y chercher les sources de la transcendance ; Paul Klee l'avait ainsi compris, qui repose sous ces mots gravés sur sa tombe : «je suis insaisissable dans l'immanence ». Comme le yogin, c'est dans son incarnation même, voire dans sa corporéité, que l'artiste puise la force de son œuvre; mais alors que celui-là s'en absente, celui-ci y demeure. Non pas à la manière du vulgaire qui en souffre ou en jouit dans l'ignorance, mais comme un improbable délivré-vivant qui ne se résout pas à renoncer au monde.

C'est ainsi que le rasa est paradoxalement à la fois simple et multiple : simple en ce qu'il consiste en une délectation spontanée de la conscience par elle-même, reposant en ellemême, rendue par une intuition directe à sa nature essentielle qu'est la béatitude ; multiple en ce qu'elle savoure son essence au sein même de l'expérience, dont elle assume l'indéfinie diversité. Est-ce que, comme l'énonce Vācaspati Miśra dans un autre contexte, c'est parce qu'il est dépourvu de la faculté de jouir que le corps subtil transmigre, à la recherche d'un corps physique de jouissance et d'expérience, dans l'attente de l'ultime délivrance ? «Le corps subtil est dénué de la faculté de jouir lorsqu'il est sans un corps à six enveloppes, lieu de jouissance: c'est pourquoi le corps subtil transmigre»(Kapani, 1993, p. 427). La multiplicité des expériences esthétiques dans la variation continue des huit rasa, structuraux, propres à la conscience humaine en ce qu'ils procèdent des huit dispositions d'esprit permanentes consubstantielles au psychisme inconscient, n'a jamais soulevé le moindre paradoxe, tant que n'a pas été posée la question métaphysique de l'essence du rasa, ni formulée la thèse de son unicité. Le Nātyaśāstra éclaircit des difficultés portant sur les conditions de l'expérience esthétique et sur la corrélation entre bhāva(sentiment) et rasa (sentiment esthétique), mais élude la question de l'essence, en expliquant simplement en quoi le premier est cause de la manifestation du second ; à la question «qu'est-ce que le rasa?» Bharata répond par un argument analogique : le rasa est à l'œuvre ce que le goût est à un plat bien cuisiné, le rasa est ce qui est susceptible d'être savouré ; à quoi bon tenter de cerner leur principe, prévient-il, sachant que l'art est un océan insondable de Connaissance, et qu'il est impossible d'en atteindre la limite?

Le travail philosophique d'Abhinavagupta affronte, peut-être mieux que d'autres avant lui, l'exigence de conceptualisation en une argumentation dont je viens d'évoquer les thèses

\footnotetext{
${ }^{74}$ Hațha-yoga-pradīpikā, I-12.
} 
principales. L'essence du rasa est la béatitude constitue non seulement la formule ontologique de l'art, mais aussi sa définition phénoménologique. Ajouterait-on la détermination d'une visée normative ? Toute expérience de plaisir ou de satisfaction est certes « comme une goutte d'eau dans l'océan de félicité », et mérite comme telle l'attention, confient les philosophes tantriques, sensibles au tout de l'existence, et Spinoza par exemple ne les démentirait pas, qui entend les passions de joie comme accroissement de la force d'exister. Mais pas plus que celui-ci n'y reconnaît la condition suffisante de la connaissance et de la liberté, Abhinavagupta ne se satisfait d'un art qui imite les affections de l'existence ordinaire : il exige une joie qui les transcende toutes. Partant, le dégoût n'est pas une affection de dégoût mais paradoxalement un affect de joie ; l'aversion commune à l'égard d'un objet que l'on juge, dans un sentiment d'intense malaise, très déplaisant, y perd sa force d'agitation avec le jugement lui-même. "La douleur, affirme le philosophe, est simplement et seulement une absence de repos [de la conscience en elle-même]»(Gnoli, p. 90). La conscience contemplative parfaitement absorbée dans la délectation de l'œuvre est prémunie contre toute forme de douleur ; simplement «colorée » par la bhāvanā qui puise sa force dans la diversité des affects latents, elle jouit de sa propre puissance. On ne saurait douter du lien implicite entre la puissance de jubilation dont l'art est porteur et le progrès spirituel du sujet. Une évocation de la théorie des bhāva dans la doctrine Śāmkhya(l'un des six courants philosophiques de l'Inde ancienne) contribuerait à l'assurer; le commentaire de Gauḍapādainsiste sur la variation des dispositions d'esprit dans les activités et leur choix dans la vie quotidienne, et distingue les bonnes (sattviques) des mauvaises (tamasiques) selon leur valeur sotériologique. Le détachement (ou dépassionnement), au même titre que la vertu, la connaissance et le pouvoir, constitue la forme lumineuse de l'intelligence, et est ainsi présenté (Kapani, op. cit., p. 433) :

«Le détachement est de deux sortes : extrinsèque, intrinsèque. Le détachement extrinsèque est l'absence d'attachement à l'égard des sens ; il est né du dégoût. Ce dégoût naît (lui-même) quand on considère les objets qu'il faut acquérir, conserver, perdre, auxquels on s'attache et par lesquels on peut être meurtri. Le détachement intrinsèque naît dans l'esprit de celui qui désire la délivrance ».

Le dégoût est décrit comme forme lumineuse de la buddhi en tant qu'il est rapporté au dépassionnement qu'il provoque; les attachements illusoires et ignorants aux objets périssables (l'ensemble du réel manifesté) sont le lot de la souffrance et de l'existence servile, dont le dégoût libère. Celui-ci ne s'interprète pas au sens d'une affection pathogène qui nous pénètre et nous enchaîne à la douleur, mais comme moment d'un procès de détachement «intrinsèque » qui nous en affranchit, en épuisant progressivement ces passions qui d'ordinaire laissent leurs impressions déterminantes dans le psychisme. Il n'est pas une affection serve, mais un affect qui délivre, accompagné d'un jugement de connaissance.

Point de tel jugement dans l'intuition artistique, où le détachement accompagne spontanément l'effacement des obstacles. Ceci d'autant plus que l'art se passe, de par sa spécificité même, de toute représentation mentale, comme c'est le cas particulièrement en musique.

\section{Prolongements}

On l'aura compris, la raison de l'activité artistique est de provoquer une expérience esthétique, qui est aussi bien spirituelle. Le matériau de la création (les mots, les gestes, les sons, etc., et plus largement les déterminants artistiques) est ce par quoi le savoir-faire de l'artiste induit (pour lui-même comme pour le contemplateur) l'émergence de sentiments d'une nature particulière, variables en nature et en intensité, sublimés de l'expérience ordinaire, éventuellement très proches de ceux auxquels conduit la méditation spirituelle, qui 
est considérée dans la culture indienne comme la pratique la plus accomplie de l'expérience humaine, culminant dans la béatitude (ānanda). A chacun, s'il le souhaite, de généraliser les fruits de cette expérience esthétique, de les prolonger jusqu'à envahir la conscience tout entière, au point d'instaurer une nouvelle manière de vivre.C'est un travail de transformation, de conversion. Une entreprise de sagesse.

Bien entendu, personne n'est tenu de le faire, le travail de l'artiste n'engage que luimême, et chacun contemple de la manière qu'il peut. Mais une simple participation à l'œuvre de l'homme ordinaire (assister à une représentation théâtrale par exemple) est déjà, au moins, une consolation des tourments et, dans le plaisir, l'expérience d'une "goutte d'eau dans un océan de félicité ».

Pour nous autres, européens, cette conception n'est pas si impénétrable qu'il pourrait sembler. Certes, les références culturelles ne sont pas les nôtres. Mais l'expérience ellemême ? N'est-ce pas, dans le fond, une enjeu possible de questionnement, voire d'éducation ? Qui, en outre, n'a jamais éprouvé cette sensation d'ineffable qui surgit parfois, irrésistible et inattendue, à la contemplation d'une œuvre particulièrement puissante ? N'est-ce pas là ce que suggère, en d'autres termes, par d'autres concepts, selon d'autres références culturelles et d'autres œuvres, un Jankélévitch ${ }^{75}$ par exemple?

En dépit des différences notables entre nos cultures, des lignes de convergence ou d'interrogation mutuelle, de mise en perspective, se dégagent.Je voudrais en suggérer quelques-unes.

En premier lieu, on ne manquera pas de noter la concordance entre l'étymologie commune de nos termes de saveur, savoir et sagesse d'un côté, et le rapprochement équivalent que font les indiens dans leur interprétation du mot rasa. Suc, saveur, le rasa est ce qui se savoure, et dans le même temps renvoie, comme catégorie philosophique, à la notion de connaissance, laquelle est inextricablement liée en Inde à la visée spirituelle, de sagesse donc. La saveur sensible de l'œuvre se prolonge en saveur de l'existence par ce que les deux expériences ont en commun dans leur accomplissement le meilleur, la jubilation ou béatitude (ānanda), que la métaphysique indienne définit comme essence du réel (brahman).La visée de sagesse de notre culture antique n'est pas si éloignée de cela - et l'on trouvera même chez Pythagore par exemple ou dans certains textes de Plotin à propos du beau, des accents assez proches.De même, la distinction entre savoir et saveur, en un premier sens, fait le partage entre ce que l'on connaît intellectuellement et ce dont on est pratiquement capable (disons les ordres respectifs du concept et de l'affect); mais en un second sens, elle les relie, dans la mesure où le rapport au savoir est savoureux, et la capacité à savourer constitue une sorte de savoir. C'est ce que désigne bien souventla notion de sagesse. On trouve la même ambiguïté dans la pensée esthétique indienne, quisuggère deux types de savoirs : celui qui s'enseigne (vyutpatti), et que la poésie ou le théâtre transmettent, non pas à la façon intellectuelle et austère d'un maître, mais par la saveur du rasa. Ce savoir, instruction portant sur le contenu culturel des textes, des représentations, reçu dans la jubilation esthétique, se prolonge en une autre forme de plaisir (prīti), en quoi consiste a posteriori la conscience joyeuse d'être instruit. Ce qui fait dire à Abhinavagupta que plaisir et instruction ne font qu'un. Mais nous avons également évoqué une autre forme de joie et de connaissance : joie de béatitude propre au rasa (forme ultime de jubilation), qui est en même temps connaissance parfaite de ce que nous sommes, parcelle d'absolu qui est félicité (ānanda). La connaissance à laquelle conduit l'expérience jubilatoire particulière de l'art (qui transmet dans le même mouvement une

\footnotetext{
${ }^{75}$ Je renvoie ici aux magnifiques ouvrages de Jankélévitch sur l'esthétique musicale, en particulier La musique et l'ineffable, Seuil, 1983.
} 
instruction culturelle, intellectuelle) sublime cette expérience en une joie parfaite, qui est aussi, dans la tradition de l'Inde, connaissance véritable.

En outre, la théorisation de la poésie et du théâtre indiens comme processus d'imitation, de représentation, voué à la sublimation ( "généralisation») par les effets de transformation des affects ordinaires en sentiments esthétiques, lesquels favorisent à leur tour la transformation des manières de vivre, pose d'une façon pour nous renouvelée la question générale des rapports entre l'art et la vie, dont la connaissance aurait sans doute beaucoup apporté, parmi mille autres, à un Rilke (Lettres à un jeune poète ${ }^{76}$ ) ou à un Proust (Sur la lecture $\left.^{77}\right)$. Probablement aussi, plus proche de nous, Paul Ricoeur aurait-il été intéressé de voir comment le travail de configuration, puis surtout de refiguration, peut conduire le lecteur (le spectateur) à découvrir, comme il le disait, des dimensions dissimulées de l'expérience humaine et de transformer sa vision du monde. A la notion de catharsis empruntée à Aristote, qu'il étudie dans un chapitre de Temps et Récit en la traduisant par les expressions de clarification, éclaircissement ou épuration ${ }^{78}$, il aurait trouvé une alternative singulière sous l'espèce de la « généralisation » de l'esthétique indienne que j'ai évoquée plus haut. Mais plus encore, il aurait peut-être été étonné de découvrirque, si l'aisthèsis aristotélicienne libère le lecteur du quotidien, si la catharsis le rend libre pour de nouvelles évaluations de la réalité prenant la forme de la relecture, et si, par un effet plus subtil encore elle «amorce un processus de transposition, non seulement affective mais cognitive ${ }^{79}$, la pensée indienne entreprend de son côté l'analyse d'un processus inversé, par lequel l'expérience esthétique convoque le cognitif pour solliciter l'affectif, et sublimer l'affectif ordinaire en affect spirituel. Une autre manière de «devenir lecteur de soi-même » (Ricoeur reprend cette expression de Proust) par cette active réorganisation de notre être-au-monde en quoi consiste le travail de refiguration.

Par ailleurs, la théorie indienne (psychologique, philosophique, esthétique) des sentiments, (bhāva), englobantnotamment les dispositions d'esprit permanentes (sthāyibhāva), temporaires (vyabhicāribhāva), et les saveurs esthétiques (rasa), et que je n'ai fait ici que survoler, mériterait d'être examinée à la lumière des travaux contemporains en neurobiologie. Peut-être Antonio Damasio (2003), étudiant une nouvelle classification des émotions et une nouvelle détermination des sentiments, aurait-il pu écrire un «Abhinavagupta avait raison »? Peut-être Rémy Lestienne (2008) se réjouirait-il de connaître les vues des penseurs indiens, en examinant l'incidence de l'expérience esthétique sur le cerveau affectif ?Et que dirait Bernard Lechevalier (2010), après ceux de Mozart et de Baudelaire, sur le cerveau des musiciens indiens?

Enfin, la méfiance que les penseurs de l'Inde ont toujours eue à l'égard des constructions intellectuelles et du travail théorique, et ce malgré la somme impressionnante de grands textes sanskrits, n'est pas sans rappeler notre tradition sceptique.On se souvient, comme je l'ai suggéré plus haut, des doutes de Montaigne à propos des élaborations philosophiques : «Je ne me persuade pas aisément qu'Épicure, Platon et Pythagore nous aient donné pour argent comptant leurs "atomes », leurs "idées » et leurs "nombres". Ils étaient trop sages pour établir leurs articles de foi de chose si incertaine et si débattable » (2002, p. 374). Les Indiens parce qu'ils considéraient l'activité intellectuelle comme cause

\footnotetext{
${ }^{76}$ Voir notamment les lettres du 17 février et du 23 avril 1903.

${ }^{77}$ Proust, Sur la lecture, Actes Sud, 1988.

${ }^{78}$ Ricoeur P., Temps et Récit, Seuil essais, 1991, T. 1, pp. 101-104.

${ }^{79}$ Ricoeur P., Temps et Récit, Seuil essais, 1991, T. 3, p. 323.
} 
d'illusions, ou au mieux comme limitée, impuissante à conduire à la connaissance absolue (béatitude propre à la méditation); les sceptiques Européens parce qu'ils doutaient que nous ayons accès à aucune certitude absolue. Hygiène d'esprit communément partagée contre tous les dogmatismes, ce qui ne les a jamais empêchés, ni les uns ni les autres, de penser aussi loin qu'ils le pouvaient.

\section{Bibliographie}

BANSAT-BOUDON L., Poétique du théâtre indien. Lectures du Nātyaśāstra, Publications de l'École Française d'Extrême-Orient, vol. 169, 1992.

BANSAT-BOUDON L. (dir.), Théâtre de l'Inde ancienne, Gallimard, 2006.

BANSAT-BOUDON L., SCHEID J. (dir.),Le disciple et ses maîtres, Seuil, 2002.

BHARATA,TheNātyaśāstra,Śri Satguru Publication, Rāga Nṛtya series, N², 1988.

BUGAULT G., L'Inde pense-t-elle ?, PUF,1994.

COOMARASWAMY A. K., The Transformation of Nature in Art, Harvard, 1934

DAMASIO A., Spinoza avait raison.Joie et tristesse, le cerveau des émotions, Odile Jacob, 2003.

DUFRENNE M., Phénoménologie de l'expérience esthétique. 2 La perception esthétique, PUF, 1953/1992.

FILLIOZAT P.-S., Le Pratāparudrīya de Vidyānātha, Publications de 1'Institut Français d'Indologie, $\mathrm{n}^{\circ} 26,1963$.

GNOLI, R.,The Aesthetic Experience According to Abhinavagupta, Istituto Italiano per il Medio ed Estremo Oriente, Serie Orientale Roma, Vol. XI, 1956. 1994.

HULIN M., Qu'est-ce que l'ignorance métaphysique (dans la pensée hindoue) ?, Vrin,

HULIN M., Le principe de l'ego dans la pensée indienne classique, Collège de France, Publications de l'Institut de civilisation indienne, fascicule 44,1978.

KAPANI L.,La notion de samskāra, Collège de France, Publications de l'Institut de civilisation indienne, fascicule 59, 2 vol (1992/1993).

LECHEVALIER B., Le cerveau mélomane de Baudelaire, Odile Jacob, 2010.

LESTIENNE R., Miroirs et tiroirs de l'âme. Le cerveau affectif, Odile Jacob, 2008.

MICHAËL T., La Symbolique des gestes des mains selon l'Abhinaya-Darpana, Sémaphore, 1985.

MASSON J. L., PATWARDANM. V., Aesthetic Rapture, the Rasādhyāya of the Nätyaśāstra,Deccan College, Postgraduate and Research Institute in Poona, 1970. 
MONIER-WILLIAMS, A Sanskrit-English Dictionnary, Delhi, Motilal Banarsidass publ., 1990.

MONTAIGNE M. de, Les Essais, Arléa, 2002.

POGGI C., «La conception esthétique d'un maître du śivaïsme cachemirien : Abhinavagupta », Adyatan, publ. Langues’O, n5, pp. 133-144, 1989.

RAJA K., The Langage of Poetry, Extension Lectures Publication Series : 29, Karnatak University, Dharwad, 1978.

SILBURN L., Le Vijñāna bhairava, Institut de Civilisation Indienne, De Boccard, 1983.

SIAUVE S., « Le guru dans la tradition hindoue », Le Maître spirituel, Cerf, 1980.

SOLERE J.L. (dir.), «L'Orient de la pensée, philosophies en Inde », Les cahiers de philosophie, $\mathrm{n}^{\circ} 14,1992$. 